\title{
Immunological Profiling of Patients with Rare Short Stature, Optic Nerve Atrophy, and Pelger-Huet Anomaly (SOPH) Syndrome
}

\author{
L.R. Zhozhikov ${ }^{1}$, F.F. Vasilev ${ }^{1, *}$, N.R. Maksimova ${ }^{1}$ \\ 1 Laboratory of Molecular Medicine and Human Genetics, North-Eastern Federal University (NEFU), Yakutsk, \\ Russia \\ *Corresponding author. Email:ff.vasilev@s-vfu.ru
}

\begin{abstract}
SOPH syndrome (SOPH; MIM\# 614800) is a rare hereditary monogenic disease caused by a mutation in the NBAS gene. The clinical spectrum of this disease affects many symptoms such as skeletal dysplasia, atrophy of the optic nerves, Pelger's leukocyte abnormality. While the somatic manifestations of this disease are known and the genetic cause has been identified, the involvement of immunological disorders in the pathogenesis of the disease remains unknown. Also, mutations in the NBAS gene are the cause of isolated recurrent liver failure, precipitated by infantile liver failure syndrome 2 (ILFS2; MIM\#616483) and other combined phenotypes. An analysis of the immunological status of patients with SOPH syndrome was carried out in order to detect disorders in cellular and humoral immunity. A significant decrease in the level of immunoglobulins (IgA, IgM, IgG, IgE) and the level of NK cells was found. These studies will help in the future to establish a reliable pathogenesis of the disease and to find approaches to the treatment of patients with disorders in the NBAS gene.
\end{abstract}

Keywords: immunological profiling, rare short stature, optic nerve atrophy, Pelger-Huet anomaly (SOPH) syndrome

\section{INTRODUCTION}

Pathogenic variant in the neuroblastoma amplified sequence $(N B A S)$ gene was described in the Yakut population as a cause of short stature, optic-nerve atrophy, and the Pelger-Huet anomaly of granulocytes (SOPH) syndrome with autosomal recessive inheritance (OMIM \#614800). Other mutations in NBAS gene have been reported to cause multisystemic disorders with wide range of phenotypes including recurrent acute liver failure, skeletal dysplasia, eyes pathologies and immunological abnormalities. Although SOPH patients developed a frequent respiratory infection, immunological parameters were not examined.

SOPH syndrome is associated with a mutation in the NBAS gene (neuroblastoma amplified sequence), located on the short arm of the second chromosome, due to the replacement of guanine for adenine (c. 5741G> A) in exon 45, which leads to the replacement of arginine for histidine (p.R1914H) in the structure of the NBAS protein. The region where this mutation takes place expresses that part of the protein that binds the NBAS protein to the RINT1 and ZW10 polypeptides that are part of the Syntaxin 18 complex; which, in turn, plays a role in the fusion of cell organelle membranes and transport between the endoplasmic reticulum and the Golgi apparatus.

The NBAS gene consists of 52 exons and contains 420 thousand base pairs. Gene abnormalities modulate the expression of 1444 human genes, which corresponds to almost $10 \%$ of all expressed genes. Some of them are associated with embryonic development and cholesterol biosynthesis, which makes research on this gene critical for science and health.

There are also studies supporting the association of NBAS protein with nonsense mediated RNA decay 
(NMD), which cleaves RNAs containing stop codons in the wrong places (in the open reading frame) and, therefore, incorrectly spliced. This system allows to control non-viable RNA from contact with ribosomes at the exit from the nucleus. It is believed that the effectiveness of NMD may be directly related to the severity of symptoms and the outcome of genetic diseases. Studies in this area are sporadic, and this function of the NBAS protein is poorly understood.

The authors of this work made an attempt to assess the immune status of patients with a rare hereditary disease SOPH syndrome. A comparative assessment was also made with similar scientific articles concerning patients with a mutation in the NBAS gene with immunodeficiency states.

\section{MATERIALS AND METHODS}

Sampling was carried out from unrelated Yakut patients with SOPH syndrome and healthy individuals. The percent and number of immunocompetent cells were determined by flow cytometry. Immunoglobulin serum levels were determined using ELISA kits. In experiments we used equipment of NEFU's Center for Collective Use.

\section{RESEARCH RESULTS}

Immunodeficiency has not been described in SOPH patients with NBAS mutations. To further extend this trait as part of the SOPH syndrome, we performed immunophenotyping in several SOPH Yakut patients and compare to few Yakut control individuals. As shown in Fig.1, the $\mathrm{T}$ and $\mathrm{B}$ cell counts was not impaired in Yakut SOPH patients while NK cell counts may be slightly reduced. Most strikingly, the relative level of the various serum immunoglobulin isotypes was diminished in the SOPH Yakut population. Interestingly, Yakut SOPH patients are prone to infections like pneumonia, bronchitis, and upper respiratory tract infection (URT). Patients with SOPH syndrome have URT 4-5 or more frequently than control population, further strengthening that NBAS mutations affect the immune system function. The mechanism by which NBAS mutations can cause an immunodeficiency remains to be elucidated, knowing that the NBAS protein is ubiquitously expressed and may be interacting with SNARE proteins involved in fusion of membranes and that its deficiency promptly leads to an ER stress response.

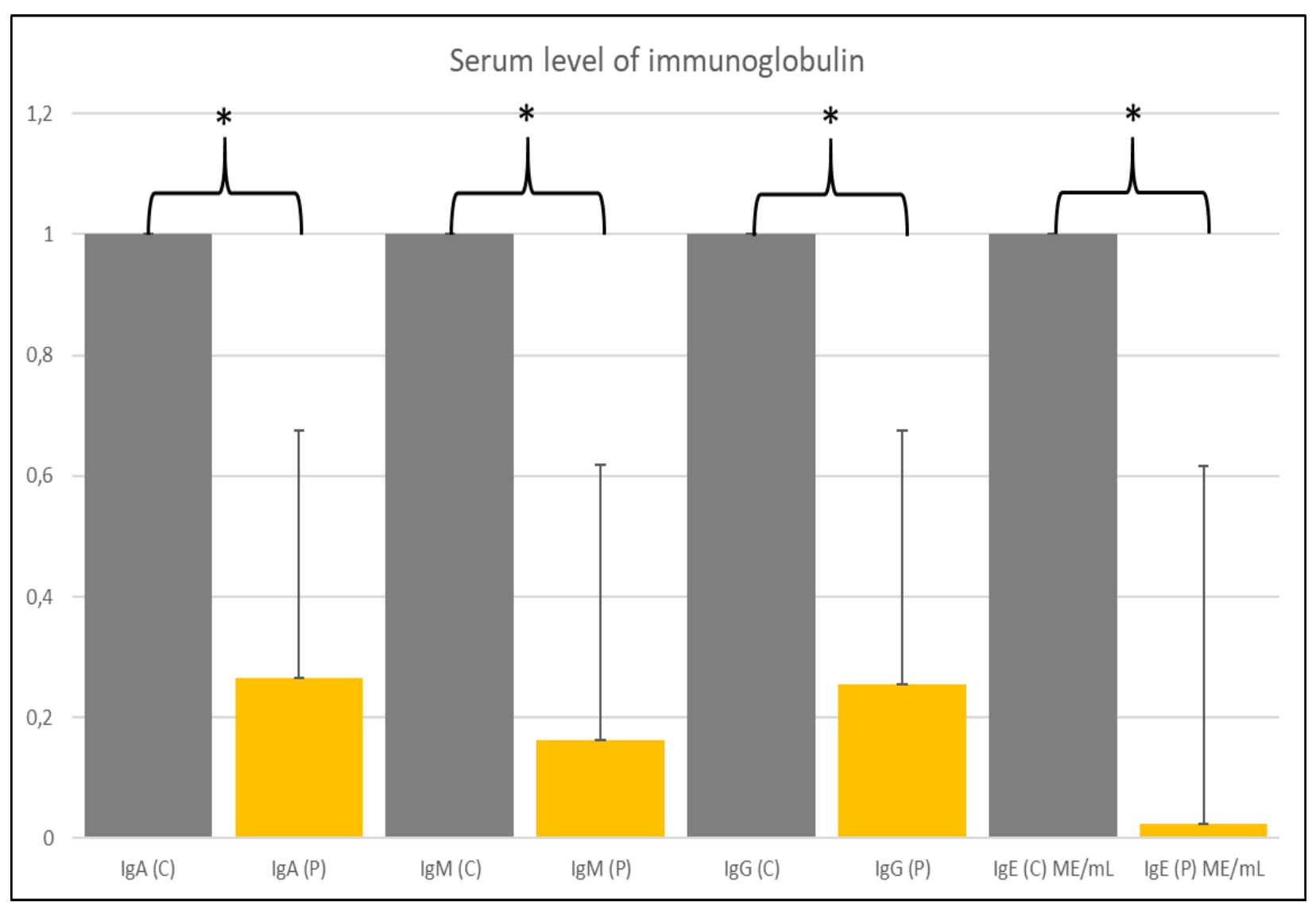

Figure 1. Serum levels of immunoglobulin content. $\mathrm{P}$ - patients, $\mathrm{C}$ - control. (data are presented as means and standard deviation) 


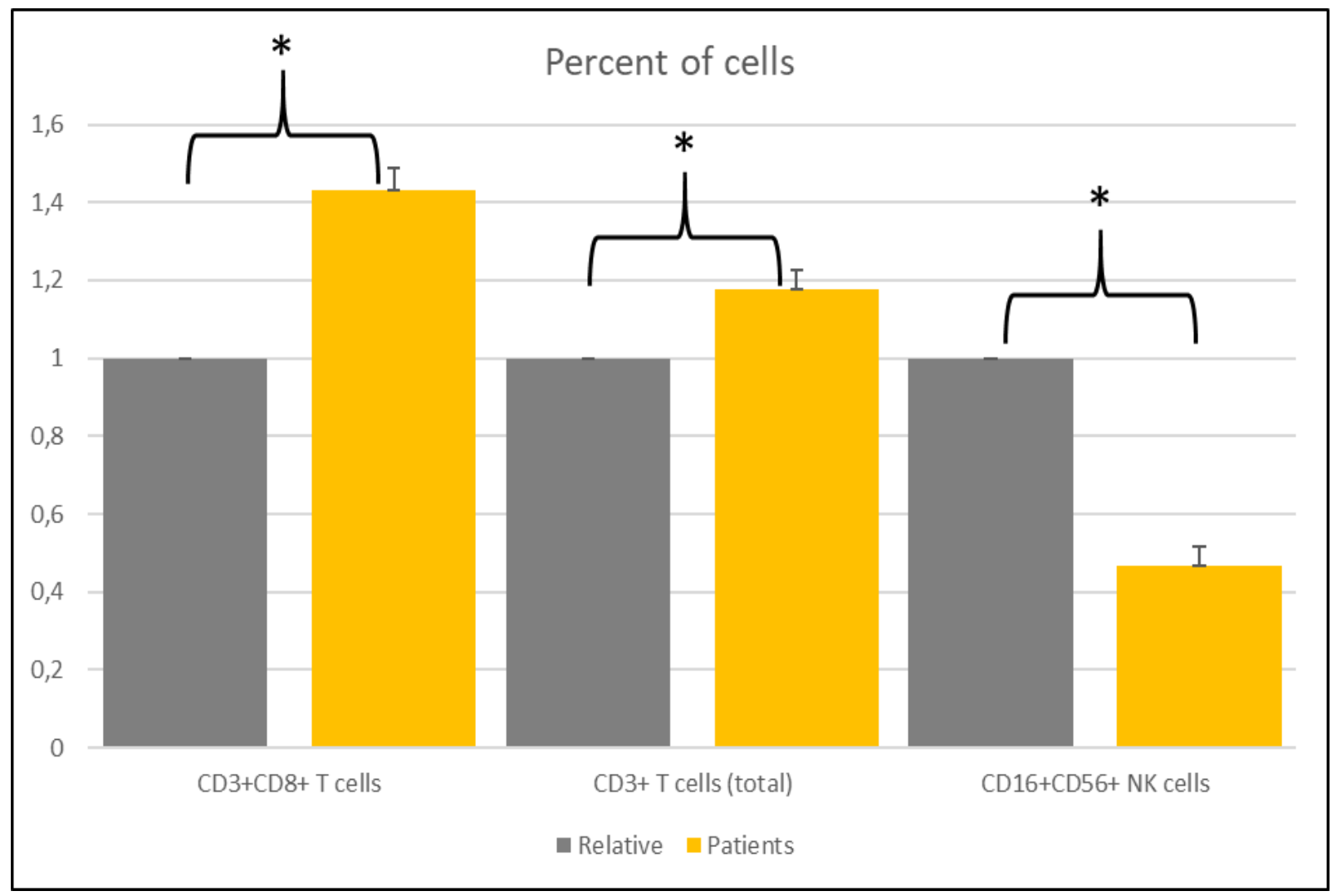

Figure 2. Percentage of immunocompetent cells. (data are presented as means and standard deviation)

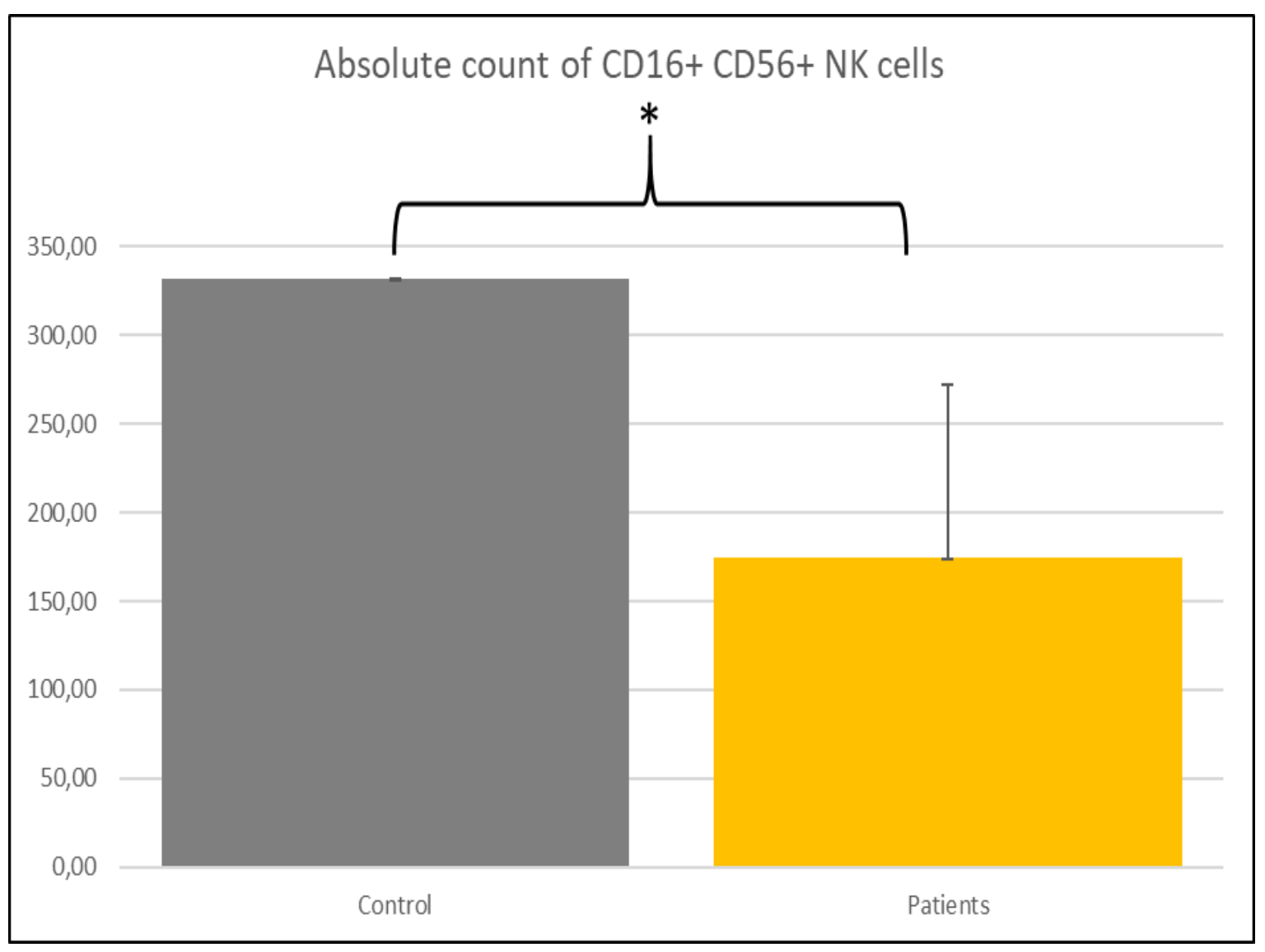

Figure 3. The absolute amount of NK-cells (data are presented as means and standard deviation) 
Serum immunoglobulins (IgA, IgM, IgG, IgE) were significantly reduced in SOPH patients in compare with controls. $\mathrm{CD}^{+}$and $\mathrm{CD}^{+}{ }^{+} \mathrm{T}$ cells amounts were unremarkable. Patients with SOPH syndrome characterized by low percentage and count of $\mathrm{CD} 16^{+} \mathrm{CD} 56^{+} \mathrm{NK}$ cells and slightly lower levels of $\mathrm{CD} 19^{+} \mathrm{B}$ cells.

\section{DISCUSSION}

At the moment, in the world literature, the authors of this work have found two scientific articles particularly related to immunodeficiency states in patients with a mutation in the NBAS gene. In the authors of one of them, the patient is a newborn from two consanguineous parents. His condition was monitored for seven months. The patient was found to have a mutation in the NBAS gene that was not previously described in the literature a homozygous variant in NBAS: c. [1948C >T], p.Pro650Ser, inherited from both parents. Serum immunoglobulin $\mathrm{G}(\mathrm{IgG})$ were markedly decreased, $\operatorname{Ig} \mathrm{A}$ and $\operatorname{IgM}$ were undetectable, and levels of $\operatorname{IgE}$ were slightly augmented. Complete absence of CD19+ cells, low count of CD8+ lymphocytes, and reduced natural killer (NK) levels was also identified.

Other variants of the immunodeficiency state were excluded. In addition to immunological symptoms (frequent infectious lesions, etc.), a triangular face, proptosis, flat groove, mild retrognathia, hirsutism, loose and slightly wrinkled skin, and a clear decrease in subcutaneous fat were also found.

The authors of the second study we found reviewed three patients with a mutation that causes SOPH syndrome (c. 5741G> A), together with deletion of exons 35-47 in two of them and an additional insertion in the third (c.1628_1629insA).

In addition to the already known symptoms of $\mathrm{SOPH}$, these patients also showed decreased amounts of immunoglobulins and a decrease in the level of natural killer cells. But it should be noted that, in contrast to patients with SOPH, the patients presented in the work of these authors have impairments in intelligence, episodes of liver failure and osteogenesis imperfecta, which was not previously observed in patients with SOPH syndrome.

\section{CONCLUSIONS}

We suggest that impaired immunological features contribute to recurrent infections in SOPH patients. We would like to emphasize that physicians should pay attention to immunodeficiency in SOPH patients to start appropriate treatment. Functional analysis of mutational impact on immunocompetent cells is essential to understand the pathophysiology of NBAS pathology.
Grant references: This work was supported by the Ministry Education and Science of Russian Federation (Project No. FSRG-2020-0014 "Genomics of Arctic: epidemiology, hereditary and pathology").

\section{REFERENCES}

[1] D. Carli et al., NBAS pathogenic variants: defining the associated clinical and facial phenotype and genotype-phenotype correlations, Hum. mutat. 40(6) (2019) 721-728.

[2] B. Jiang et al., Case Report: Pediatric Recurrent Acute Liver Failure Caused by Neuroblastoma Amplified Sequence (NBAS) Gene Mutations, Front. in Pediatr. 8 (2020).

[3] A. Khoreva et al., Complex Multisystem Phenotype with Immunodeficiency Associated With NBAS Mutations: Reports of Three Patients and Review of the Literature, Front. in pediatr. 8 (2020) 577.

[4] D. Longman et al., Identification of a localized nonsense-mediated decay pathway at the endoplasmic reticulum, Gen. \& developm. 34(1516) (2020) 1075-1088.

[5] N. Maksimova et al., Neuroblastoma amplified sequence gene is associated with a novel short stature syndrome characterised by optic nerve atrophy and Pelger-Huët anomaly, J. of med. genet. 47(8) (2010) 538-548.

[6] D.A. Petukhova et al., Identification of a Novel Compound Heterozygous Variant in NBAS Causing Bone Fragility by the Type of Osteogenesis Imperfecta, International Symposium on Bioinformatics Research and Applications, Springer, Cham, 2020, pp. 38-43.

[7] S. Ricci et al., Immunological features of neuroblastoma amplified sequence deficiency: report of the first case identified through newborn screening for primary immunodeficiency and review of the literature, Front. in immunol. 10 (2019) 1955.

[8] C. Staufner et al., Defining clinical subgroups and genotype-phenotype correlations in NBASassociated disease across 110 patients, Genet. in Med. 2 (2020).

[9] J.E. Yu, J.S. Orange, Y.Y. Demirdag, New primary immunodeficiency states: current state and prospects, Pediatr. named after G.N. Speransky 98(3) (2019). 